\title{
Age and gender differences in clinical outcomes of patients with heavy-calcified coronary artery lesions treated percutaneously with rotational atherectomy
}

\author{
Rafał Januszek B,2,A-F, Artur Pawlik',B, Bartłomiej Staszczak ${ }^{3, B}$, Magdalena Jędrychowska ${ }^{2, B}$, \\ Jerzy Bartuś3, , Jacek Legutko ${ }^{4, E}$, Dariusz Dudek ${ }^{2,5, \mathrm{E}, \mathrm{F}}$, Andrzej Surdacki ${ }^{2,6, \mathrm{E}}$, Stanisław Bartuśs ${ }^{2,6, \mathrm{E}, \mathrm{F}}$ \\ ${ }^{1}$ University of Physical Education, Department of Clinical Rehabilitation, Kraków, Poland \\ 2 Department of Cardiology and Cardiovascular Interventions, University Hospital, Kraków, Poland \\ 3 Jagiellonian University Medical College, Kraków, Poland \\ ${ }^{4}$ Institute of Cardiology, Jagiellonian University Medical College, Kraków, Poland \\ ${ }^{5}$ Department of Interventional Cardiology, Jagiellonian University Medical College, Kraków, Poland \\ ${ }^{6}$ Department of Cardiology, Jagiellonian University Medical College, Kraków, Poland \\ A - research concept and design; B - collection and/or assembly of data; $C$ - data analysis and interpretation; \\ $\mathrm{D}$ - writing the article; $\mathrm{E}$ - critical revision of the article; $\mathrm{F}$ - final approval of the article
}

Address for correspondence

Rafał Januszek

E-mail: jaanraf@interia.pl

Funding sources

None declared

Conflict of interest

None declared

Received on February 8, 2019

Reviewed on April 29, 2019

Accepted on June 27, 2019

Published online on February 19, 2020

Cite as

Januszek R, Pawlik A, Staszczak B, et al. Age and gender differences in clinical outcomes of patients with heavycalcified coronary artery lesions treated percutaneously with rotational atherectomy. Adv Clin Exp Med. 2020;29(2):225-233. doi:10.17219/acem/110314

D0I

10.17219/acem/110314

Copyright

Copyright by Author(s)

This is an article distributed under the terms of the

Creative Commons Attribution 3.0 Unported (CC BY 3.0)

(https://creativecommons.org/licenses/by/3.0/)

\section{Abstract}

Background. Rotational atherectomy (RA) used in elderly patients treated with percutaneous coronary interventions (PCI) could enable revascularization or the omission of cardiac surgery. Knowledge about factors affecting the prognosis may improve the results of treatment.

Objectives. We aimed to assess the relationship of gender and age with long-term clinical outcomes expressed as major adverse cardiac and cerebrovascular events (MACCEs).

Material and methods. The study included 97 consecutive patients treated with PCl and RA at the mean age of 71 . The study group contained $73.2 \%$ men and $26.8 \%$ women, $36.1 \%$ of patients older than 75 and $63.9 \%$ younger than 75 . The mean time of follow-up was $695.3 \pm 560.9$ days. The rate of MACCEs (deaths, myocardial infarctions (MIs), reinterventions, coronary artery by-pass surgeries, or cerebral strokes (CSs)/ transient ischemic attacks (TIAs)) in the overall group of patients was calculated at 33.7\%.

Results. The comparison of Kaplan-Meier survival curves did not depict significant differences in the frequency of MACCEs for age $(p=0.36)$ and gender $(p=0.07)$. We noticed that the death rate was higher in females than in males and in patients older than 75 compared to those younger, and was statistically significant for age $(p=0.04)$. The rate of periprocedural complications was significantly higher among women than among men ( $p=0.005$ ) and in patients older than 75 compared to the younger ones $(p=0.003)$.

Conclusions. Age and gender are not significantly associated with an increased rate of MACCEs during follow-up in elderly patients treated with PCl and RA.

Key words: age, predictors, gender, clinical outcomes, rotational atherectomy 


\section{Introduction}

In the era of population aging and the increase in the percentage of elderly patients, differences in the therapeutic approach among this group of patients are of special importance. A number of published studies have shown that age is associated with increased mortality and worse prognosis in the follow-up period among hospitalized patients. ${ }^{1}$ Poorer prognosis in the elderly is associated with several factors reflecting the functional changes in their organisms. Some researchers even attempted to identify the most important independent risk factors and created a prognostic index for this group of patients to estimate the probability of mortality, and thus, the possibility of interfering with these factors to prolong life in this group of patients. ${ }^{2}$ One of the independent risk factors of increased mortality in patients after hospitalization is ischemic heart disease. Previously published studies have shown differences in response to the established treatment regimens in the elderly group of patients, and that appropriate modification of the diagnostic and therapeutic processes in this group of patients may contribute to a significant improvement in treatment outcomes compared to younger patients. ${ }^{3,4}$ Increased calcification in coronary arteries is associated with a higher risk of ischemic heart disease and cardiovascular events as well as mortality in elderly patients. ${ }^{4}$ Rotational atherectomy (RA) as a device to facilitate percutaneous coronary interventions (PCI) is dedicated to patients with heavy calcified stenoses in the coronary arteries. ${ }^{5}$ The RA is applicable to elderly patients, although the number of publications comparing the results of its use depending on age is limited. Several studies have shown that age is an independent risk factor for major adverse coronary events in this group of patients. ${ }^{6}$ In comparative studies, the relationship of gender and long-term treatment results in the case of orbital atherectomy in the group of patients with heavy calcified lesions in coronary arteries has not been demonstrated, while the number of publications on RA is very limited. ${ }^{7}$

Therefore, in the current study, we aimed to assess the relationship of gender and age with long-term clinical outcomes in patients treated with PCI and RA expressed as major adverse cardiac and cerebrovascular events (MACCE).

\section{Material and methods}

Our study was a retrospective and partially prospective registry of 97 consecutive patients undergoing PCI with RA from 2002 to 2017 in the primary reference center. The majority of patients were included during the last 10 years. All patients were screened for risk factors, concomitant diseases, past PCI history, and medications. Procedural details, and in-hospital and long-term complications were collected. The maximum follow-up period was 2,315 days and the study endpoints were the rates of MACCE defined as the following: death (overall mortality), the requirement of target lesion revascularization expressed as endovascular reintervention or coronary artery by-pass grafting (CABG) operation, myocardial infarction (MI), and cerebral stroke (CS)/transient ischemic attack (TIA). All patients gave informed consent for the procedure. The study was approved by the institutional ethical board and conformed to the ethical principles for medical research involving human subjects of the 1975 Declaration of Helsinki.

\section{Procedure}

Patients were qualified for the procedure according to the current guidelines. ${ }^{5}$ A Rotablator (Boston Scientific, Marlborough, USA) was used to perform the procedure. We evaluated the preprocedural risk with Euroscore II and Syntax score online calculators (http://euroscore.org/calc. html and http://www.syntaxscore.com/calculator/start. htm respectively). The risk was defined as: low $(\leq 2 \%)$, intermediate $(3-5 \%)$ and high $(\geq 5 \%)$ for the EUROSCORE II and low (0-22 points), intermediate (23-32 points) and high ( $\geq 33$ points) for the SYNTAX score.

\section{Procedure-related complications}

The rate of procedure-related complications consisted of the intraprocedural complications and periprocedural hospitalization, which occurred after the procedure until discharge from the hospital. The procedure-related MI was defined according to the $3^{\text {rd }}$ universal definition of MI. ${ }^{8}$ Coronary artery perforation was defined according to the most common and recognized definition proposed by Ellis at al. ${ }^{9}$ The procedure-related hematoma was included in the analysis when it demanded surgical intervention or/and blood products transfusion. Cardiogenic shock was defined according to the current European Society Guidelines. ${ }^{10}$ We included all the deaths that occurred during the procedure or after the procedure until discharge from the hospital into the current analysis as overall mortality. The allergic reaction was found to be significant when it demanded prolonged hospitalization and parenteral or oral therapy with antiallergic specifics. Contrast-induced nephropathy was recognized according to the definition published by Mehran et al. ${ }^{11}$ Coronary artery dissection was defined according to the most common classification. ${ }^{12}$ Other technical complications associated with the RA procedure itself were reported in accordance with current recommendations regarding the use of RA in Europe.$^{13}$ For periprocedural arrhythmias, we included persistent ventricular and supraventricular tachyarrhythmias, and atrioventricular conductive disorders requiring additional pharmacological or interventional treatment (pacing or cardioversion). Slow-flow or no-reflow was defined as TIMI (thrombolysis in myocardial infarction) flow grade 0 or $1 .{ }^{14}$ 


\section{Statistical analysis}

Normal distribution was assessed with the Shapiro-Wilk test. The $X^{2}$ test was used to compare the categorical variables. For comparisons of continuous data, the MannWhitney U test or the Student's t-test were performed where applicable. The log-rank test was performed for comparisons of Kaplan-Meier survival curves in selected subgroups. Univariate analysis of selected potential predictors of clinical outcomes defined as MACEEs was also performed. A probability of $\mathrm{p}<0.05$ was considered statistically significant. The statistical analyses were conducted with STATISTICA v. 10.0 software (StatSoft, Inc., Tulsa, USA) and SPSS Statistics v. 24 (IBM Corp., Armonk, USA).

\section{Results}

The overall group of patients included 97 subjects in which successful rotablation was performed. Among them, there were 71 males (73.2\%) and 26 females (26.8\%), 62 individuals older than $75(63.9 \%)$ and 35 patients younger than 75 (36.1\%). The distribution of patients within particular age groups depending on gender is presented in Fig. 1.

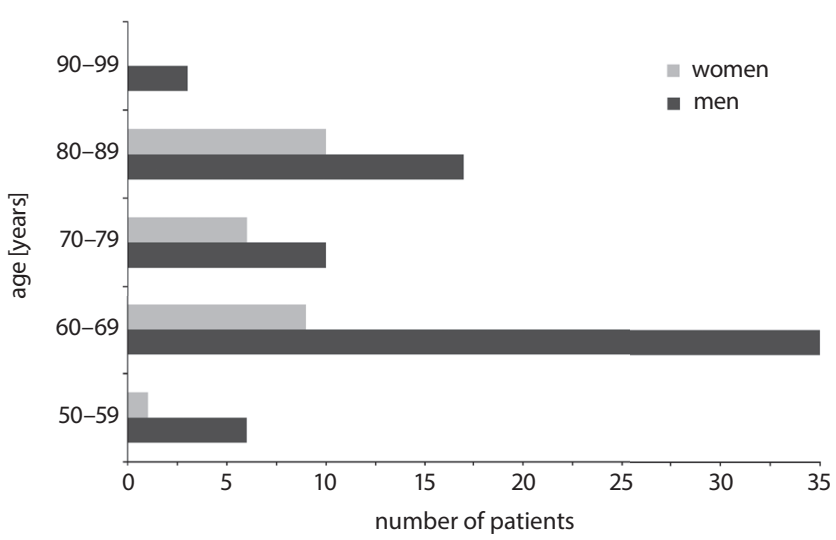

Fig. 1. Patient distribution in selected age intervals according to gender

\section{General characteristics}

The mean age of the males was $70.8 \pm 10$ years and females $73.8 \pm 9.2$ years. Males were significantly more often smokers $(69.6 \%$ vs $10 \%, \mathrm{p}=0.03)$ and their mean ejection fraction of the left ventricle (LVEF) was smaller $(43.9 \%$ vs $51.4 \%, \mathrm{p}=0.036$ ), while the only 2 cases qualified for the RA in salvage mode were females $(\mathrm{p}=0.01)$.

Patients older than 75 had significantly smaller mean body mass index (BMI) values $\left(26.7 \mathrm{~kg} / \mathrm{m}^{2}\right.$ vs $29.2 \mathrm{~kg} / \mathrm{m}^{2}$; $\mathrm{p}=0.04)$, and presented at admission significantly more often with unstable angina (UA; $28.3 \%$ vs $53.1 \%, \mathrm{p}=0.019$ ) or non-significantly less often with stable angina and nonST segment elevation MI (NSTEMI). They also were qualified significantly more often for the procedure in emergency mode (26.5\% vs 6.9\%, $\mathrm{p}=0.002)$ and non-significantly less often in elective and urgent mode. Moreover, the older group of patients characterized with greater mean serum creatinine level $(p=0.005)$ and smaller glomerular filtration rate $($ GFR) level $(\mathrm{p}<0.001)$. All clinical data are presented in Table 1.

The mean EUROSCORE II value was higher in females compared to males; however, it was without statistical significance $(3.3 \% \pm 3.3 \%$ vs $9.4 \% \pm 18.4 \%, \mathrm{p}=0.4)$. The higher mean EUROSCORE II value was attributed to the fact that among females there were 2 extremely high-risk patients treated in salvage mode, which increased the mean value. A similar relation was found when the overall group was divided according to age, where the 2 patients were placed in the group of patients older than 75 . The difference in the mean EUROSCORE II value between patients younger and older than 75 was significant $(9.2 \% \pm 15.2 \%$ vs $2.3 \% \pm 2.8 \%, \mathrm{p}<0.001)$. In the case of gender division, males were more often at low risk (44.6\%) compared to moderate (35.4\%) and high-risk patients $(0.2 \%)$, and in comparison to women, each risk group did not differ significantly. Among women, the distribution in the low (34.8\%), moderate $(34.8 \%)$ and high-risk (30.4\%) cases was more even.

In patients younger than 75 , there were more patients in the low-risk group (58.2\%) in comparison to moderate (50.9\%) and high-risk (9.1\%) groups. Not surprisingly, opposite distribution was shown in patients older than 75 , among whom most patients were in the high-risk group (45.4\%), while in the moderate (39.4\%) and low-risk (15.1\%) groups, their number was smaller. The difference between younger and older patients was significant for the low-risk group ( $\mathrm{p}=0.001)$ and high-risk group $(\mathrm{p}<0.001)$.

\section{Pharmacotherapy}

There were no statistically significant differences in pharmacological treatment between males and females. Patients older than 75 were treated significantly more often with clopidogrel as a second antiplatelet drug (83\% vs $100 \%, \mathrm{p}=0.01$ ). This was mainly due to more frequent use of ticagrelor $(8.3 \%$ vs $0 \%, \mathrm{p}=0.08)$ and prasugrel $(6.7 \%$ vs $0 \%, p=0.12$ ) in younger patients. The frequency of other medications did not differ significantly between patients younger and older than 75 . This is presented in Table 2 .

\section{Lesion characteristics and procedural indices}

The mean SYNTAX Score I value did not differ significantly between males and females, and was greater in females $(24.1 \pm 13$ points vs $26.3 \pm 13.0$ points, $\mathrm{p}=0.45)$. Patients older than 75 were presented with greater mean SYNTAX Score I value compared to younger patients; however, the difference did not reach statistical significance $(28.1 \pm 13.4$ points vs $22.1 \pm 13$ points, $\mathrm{p}=0.06)$. When comparing SYNTAX Score I groups, there were 
Table 1. General characteristics

\begin{tabular}{|c|c|c|c|c|c|c|}
\hline \multirow{2}{*}{ Variable } & \multicolumn{2}{|c|}{ Gender } & \multirow{2}{*}{$p$-value } & \multicolumn{2}{|c|}{ Age [years] } & \multirow{2}{*}{$p$-value } \\
\hline & male & female & & $<75$ & $\geq 75$ & \\
\hline Age [years] & $70.8 \pm 10.0$ & $73.8 \pm 9.2$ & 0.13 & $65.4 \pm 5.8$ & $82.6 \pm 4.0$ & $<0.000$ \\
\hline BMI $\left[\mathrm{kg} / \mathrm{m}^{2}\right]$ & $27.9 \pm 6.3$ & $28.9 \pm 4.6$ & 0.50 & $29.2 \pm 7.0$ & $26.7 \pm 3.1$ & 0.04 \\
\hline Gender (males) & - & - & & $49(79)$ & $22(62.8)$ & 0.08 \\
\hline Diabetes & $34(49.3)$ & $15(60)$ & 0.36 & $34(56.7)$ & $15(44.1)$ & 0.31 \\
\hline Hypertension & $66(95.6)$ & $25(100)$ & 0.29 & $57(95)$ & $34(100)$ & 0.68 \\
\hline Hyperlipidemia & $65(94.2)$ & $25(100)$ & 0.22 & $57(95)$ & $33(97.1)$ & 0.86 \\
\hline Obesity & $18(27.3)$ & $6(26.1)$ & 0.91 & $18(32.1)$ & $6(18.2)$ & 0.27 \\
\hline Prior $\mathrm{PCl}$ & $47(73.4)$ & $13(61.9)$ & 0.31 & $41(75.9)$ & $19(61.3)$ & 0.26 \\
\hline Prior Ml & $46(66.7)$ & $15(65.2)$ & 0.92 & $32(59.2)$ & $25(73.5)$ & 0.28 \\
\hline Prior CABG & $10(14.7)$ & $4(16)$ & 0.87 & 11 (18.6) & $3(8.8)$ & 0.43 \\
\hline PAD & $19(27.9)$ & $3(12)$ & 0.11 & $16(27.1)$ & $6(17.6)$ & 0.44 \\
\hline COPD & $9(13)$ & $5(20)$ & 0.40 & $7(16.7)$ & $7(20.6)$ & 0.24 \\
\hline $\mathrm{CS} / \mathrm{TIA}$ & $10(14.5)$ & $4(16)$ & 0.85 & $5(8.3)$ & $9(26.5)$ & 0.14 \\
\hline Heart failure & $23(33.3)$ & $8(32)$ & 0.90 & $19(31.7)$ & $12(35.3)$ & 0.72 \\
\hline Smoking & $48(69.6)$ & $10(40)$ & 0.03 & $38(63.3)$ & $20(58.8)$ & 0.71 \\
\hline Kidney failure & $13(18.8)$ & $5(20)$ & 0.93 & $8(13.3)$ & $10(29.4)$ & 0.06 \\
\hline $\begin{array}{l}\text { Clinical picture } \\
\text { stable angina } \\
\text { UA } \\
\text { NSTEMI } \\
\text { STEMI }\end{array}$ & $\begin{array}{l}36(54.5) \\
17(25.7) \\
13(19.7) \\
3(4.5)\end{array}$ & $\begin{array}{c}9(36) \\
8(32) \\
7(28) \\
1(4)\end{array}$ & $\begin{array}{l}0.11 \\
0.55 \\
0.39 \\
0.90\end{array}$ & $\begin{array}{c}32(53.3) \\
17(28.3) \\
9(15.0) \\
2(1.7)\end{array}$ & $\begin{array}{c}14(43.7) \\
17(53.1) \\
1(3.1) \\
0(0)\end{array}$ & $\begin{array}{c}0.38 \\
0.019 \\
0.08 \\
0.29\end{array}$ \\
\hline $\begin{array}{l}\text { Mode } \\
\text { elective } \\
\text { urgent } \\
\text { emergency } \\
\text { salvage }\end{array}$ & $\begin{array}{c}39(56.5) \\
21(30.4) \\
9(13) \\
0(0)\end{array}$ & $\begin{array}{l}10(40) \\
9(36) \\
4(16) \\
2(8)\end{array}$ & $\begin{array}{l}0.15 \\
0.60 \\
0.71 \\
0.01\end{array}$ & $\begin{array}{c}35(60.3) \\
21(36.2) \\
4(6.9) \\
0(0)\end{array}$ & $\begin{array}{l}14(41.2) \\
9(26.5) \\
9(26.5) \\
2(5.9)\end{array}$ & $\begin{array}{c}0.10 \\
0.39 \\
0.002 \\
0.06\end{array}$ \\
\hline LVEF [\%] & $43.9 \pm 12.7$ & $51.4 \pm 10.8$ & 0.036 & $47.8 \pm 11.5$ & $42.3 \pm 13.9$ & 0.13 \\
\hline $\mathrm{GFR}[\mathrm{mL} / \mathrm{min}]$ & $80.0 \pm 33.2$ & $64.2 \pm 29.4$ & 0.08 & $92.4 \pm 28.4$ & $48.9 \pm 17.6$ & $<0.000$ \\
\hline Creatinine $[\mu \mathrm{mol} / \mathrm{L}]$ & $92.5 \pm 33.3$ & $85.6 \pm 25.7$ & 0.21 & $83.6 \pm 20.5$ & $103.2 \pm 42.0$ & 0.005 \\
\hline
\end{tabular}

BMI - body mass index; CABG - coronary artery by-pass grafting; COPD - chronic obstructive pulmonary disease; CS - cerebral stroke; GFR - glomerular filtration rate; LVEF - left ventricle ejection fraction; MI - myocardial infarction; NSTEMI - non ST-segment elevation MI; PAD - peripheral artery disease; $\mathrm{PCl}$ - percutaneous coronary intervention; STEMI - ST-segment elevation MI; TIA - transient ischemic attacks; UA - unstable angina.

Table 2. Pharmacotherapy at discharge

\begin{tabular}{|c|c|c|c|c|c|c|}
\hline \multirow{2}{*}{ Variable } & \multicolumn{2}{|c|}{ Gender } & \multirow{2}{*}{$p$-value } & \multicolumn{2}{|c|}{ Age [years] } & \multirow{2}{*}{$p$-value } \\
\hline & male & female & & $<75$ & $\geq 75$ & \\
\hline ASA & $71(100)$ & $26(100)$ & - & $62(100)$ & $35(100)$ & - \\
\hline $\begin{array}{l}\text { Antiplatelets } \\
\text { clopidogrel } \\
\text { ticagrelor } \\
\text { prasugrel } \\
\text { ticlopidine }\end{array}$ & $\begin{array}{c}59(88) \\
4(6) \\
4(6) \\
1(1.5)\end{array}$ & $\begin{array}{c}25(96.2) \\
1(3.8) \\
0(0) \\
0(0)\end{array}$ & $\begin{array}{l}0.18 \\
0.69 \\
0.20 \\
0.53\end{array}$ & $\begin{array}{c}50(83) \\
5(8.3) \\
4(6.7) \\
1(1.7)\end{array}$ & $\begin{array}{c}34(97.1) \\
0(0) \\
0(0) \\
0(0)\end{array}$ & $\begin{array}{l}0.01 \\
0.08 \\
0.12 \\
0.44\end{array}$ \\
\hline Oral antidiabetic & 18 (26.1) & $6(25)$ & 0.93 & $21(35)$ & $3(9.1)$ & 0.03 \\
\hline Insulin therapy & $18(26.1)$ & $8(32)$ & 0.66 & $18(30)$ & $8(23.5)$ & 0.60 \\
\hline ACEis/ARB & $33(47.8)$ & $10(41.7)$ & 0.65 & $27(45)$ & $16(48.5)$ & 0.78 \\
\hline Diuretic & $34(55.7)$ & $10(50)$ & 0.95 & $23(45.1)$ & $21(70)$ & 0.28 \\
\hline$\beta$-blocker & $64(92.7)$ & $24(100)$ & 0.59 & 56 (93.3) & $32(97)$ & 0.77 \\
\hline Statin/fibrate & $68(98.5)$ & $23(95.8)$ & 0.91 & 57 (96.6) & $34(100)$ & 0.89 \\
\hline Anticoagulant & $14(20.3)$ & $6(25)$ & 0.72 & $8(13.3)$ & $12(36.4)$ & 0.06 \\
\hline Nitrate & $4(5.8)$ & $2(8.3)$ & 0.85 & $2(3.3)$ & $4(12.1)$ & 0.48 \\
\hline Ca-blocker & $20(29)$ & $9(37.5)$ & 0.35 & $19(31.7)$ & $10(30.3)$ & 0.91 \\
\hline
\end{tabular}

ASA - acetyl-salicylic acid; ACEi - angiotensin-converting enzyme inhibitors; ARB - aldosterone receptor blockers. 
no significant differences between males and females in the low $(45.6 \%$ vs $35.7 \%, \mathrm{p}=0.51)$, intermediate $(28.2 \%$ vs $28.6 \%, p=0.98)$ and advanced lesions $(26.1 \%$ vs $35.7 \%$, $\mathrm{p}=0.48$ ). While considering patients in terms of age, there were significantly more patients with less advanced atherosclerotic lesions in the coronary arteries among patients younger than 75 compared to older patients $(54.3 \%$ vs $28 \%$, $\mathrm{p}=0.04)$, whereas there was not a significantly greater percentage of patients older than 75 in the intermediate ( $36 \%$ vs $22.8 \%, \mathrm{p}=0.26$ ) and high (36\% vs $22.8 \%, \mathrm{p}=0.26$ ) SYNTAX Score I group compared to younger patients.

Both in the case of the division into women and men and into patients over and under the age of 75, atherosclerotic lesions in type $\mathrm{C}$ according to American Heart Association (AHA) classification were more frequent in comparison to type $2 \mathrm{~B}$ lesions. Type $\mathrm{C}$ lesions occurred not significantly more often in males than in females $(78.7 \%$ vs $71.4 \%, \mathrm{p}=0.56)$, and in patients older than 75 (84\% vs $73.5 \%, \mathrm{p}=0.33$ ), while type $2 \mathrm{~B}$ lesions occurred more often in females $(28.6 \%$ vs $21.3 \%, \mathrm{p}=0.56)$ and patients younger than 75 (26.5\% vs $16 \%, \mathrm{p}=0.33)$.

Females were treated significantly more often from right radial access compared to males ( $50 \%$ vs $25.9 \%, \mathrm{p}=0.04$ ), while patients older than 75 were treated significantly less often with single stent implantation compared to younger patients ( $80 \%$ vs $60 \%, \mathrm{p}=0.03)$, and insignificantly more often with 2 or 3 stents. Lesion characteristics and procedural indices are presented in Table 3.

Significantly more procedure-related complications occurred in females compared to males $(46.1 \%$ vs $18.3 \%$, $\mathrm{p}=0.005)$. Also, older patients ( $>75$ years) demonstrated a significantly higher frequency of procedure-related complications ( $42.8 \%$ vs $16.1 \%, \mathrm{p}=0.003)$. More detailed characteristics are presented in Table 4.

Table 3. Lesion characteristics and procedural indices

\begin{tabular}{|c|c|c|c|c|c|c|}
\hline \multirow{2}{*}{ Variable } & \multicolumn{2}{|c|}{ Gender } & \multirow{2}{*}{ p-value } & \multicolumn{2}{|c|}{ Age [years] } & \multirow{2}{*}{$\mathrm{p}$-value } \\
\hline & male & female & & $<75$ & $\geq 75$ & \\
\hline $\begin{array}{l}\text { Direct indication was inability to: } \\
\text { cross with b.c. } \\
\text { dilate with b.c. } \\
\text { stent delivery }\end{array}$ & $\begin{array}{c}27(40.3) \\
39(58.2) \\
1(1.4)\end{array}$ & $\begin{array}{c}8(34.8) \\
12(52.2) \\
3(13)\end{array}$ & $\begin{array}{l}0.63 \\
0.61 \\
0.02\end{array}$ & $\begin{array}{c}21(36.2) \\
34(58.6) \\
3(5.2)\end{array}$ & $\begin{array}{c}14(43.7) \\
17(53.1) \\
1(3.1)\end{array}$ & $\begin{array}{l}0.48 \\
0.61 \\
0.65\end{array}$ \\
\hline Mean burr diameter [mm] & $1.49 \pm 0.20$ & $1.43 \pm 0.30$ & 0.26 & $1.47 \pm 0.20$ & $1.47 \pm 0.20$ & 0.57 \\
\hline Maximum burr diameter [mm] & $1.5 \pm 0.20$ & $1.4 \pm 0.20$ & 0.27 & $1.48 \pm 0.20$ & $1.48 \pm 0.30$ & 0.69 \\
\hline $\begin{array}{l}\text { Arteries } \\
1 \\
2 \\
3\end{array}$ & $\begin{array}{c}56(78.9) \\
12(16.9) \\
3(4.2)\end{array}$ & $\begin{array}{c}16(64) \\
7(28) \\
2(8)\end{array}$ & $\begin{array}{l}0.13 \\
0.23 \\
0.46\end{array}$ & $\begin{array}{c}49(80.3) \\
10(16.4) \\
2(3.3)\end{array}$ & $\begin{array}{c}23(65.7) \\
9(25.7) \\
3(8.6)\end{array}$ & $\begin{array}{l}0.11 \\
0.26 \\
0.46\end{array}$ \\
\hline $\begin{array}{l}\text { Stents } \\
1 \\
2 \\
3 \\
4\end{array}$ & $\begin{array}{c}36(54.5) \\
21(31.8) \\
8(12.1) \\
1(1.5)\end{array}$ & $\begin{array}{l}15(60) \\
7(28) \\
2(8) \\
1(3.6)\end{array}$ & $\begin{array}{l}0.63 \\
0.72 \\
0.57 \\
0.48\end{array}$ & $\begin{array}{c}49(80) \\
10(16.4) \\
2(3.3) \\
0(0)\end{array}$ & $\begin{array}{c}21(60) \\
10(28.6) \\
4(11.4) \\
0(0)\end{array}$ & $\begin{array}{l}0.03 \\
0.15 \\
0.11 \\
-\end{array}$ \\
\hline Stent length [mm] & $26.2 \pm 10.2$ & $24.0 \pm 8.5$ & 0.51 & $26.6 \pm 11.0$ & $24.1 \pm 7.6$ & 0.49 \\
\hline Stent diameter [mm] & $3.04 \pm 0.70$ & $3.06 \pm 0.80$ & 0.90 & $3.05 \pm 0.70$ & $3.0 \pm 0.70$ & 0.62 \\
\hline Balloon diameter before $\mathrm{PCI}[\mathrm{mm}]$ & $2.9 \pm 0.5$ & $2.6 \pm 0.6$ & 0.06 & $2.9 \pm 0.6$ & $2.7 \pm 0.5$ & 0.27 \\
\hline Post-dilatation & $58(87.9)$ & $19(76)$ & 0.38 & $51(89.5)$ & $26(76.5)$ & 0.30 \\
\hline Balloon diameter after $\mathrm{PCI}$ [mm] & $3.6 \pm 0.7$ & $3.7 \pm 1.0$ & 0.93 & $3.6 \pm 0.7$ & $3.6 \pm 0.9$ & 0.50 \\
\hline IVUS after $\mathrm{PCl}$ & $18(25.3)$ & $3(11.5)$ & 0.29 & $15(24.2)$ & $6(17.1)$ & 0.56 \\
\hline Radiation dose [Gy] & $1.97 \pm 1.20$ & $1.9 \pm 1.2$ & 0.77 & $2.04 \pm 1.40$ & $1.8 \pm 0.8$ & 0.89 \\
\hline Contrast volume [mL] & $271 \pm 106$ & $303 \pm 140$ & 0.53 & $285 \pm 140$ & $272.0 \pm 69.6$ & 0.69 \\
\hline $\begin{array}{l}\text { Type of PCl } \\
\text { POBA/DEB } \\
\text { DES } \\
\text { BMS }\end{array}$ & $\begin{array}{c}3(4.4) \\
64(94.1) \\
1(1.5)\end{array}$ & $\begin{array}{c}0(0) \\
24(96) \\
1(4)\end{array}$ & $\begin{array}{l}0.28 \\
0.72 \\
0.45\end{array}$ & $\begin{array}{c}3(5.2) \\
55(94) \\
0(0)\end{array}$ & $\begin{array}{c}0(0) \\
33(94.3) \\
2(5.7)\end{array}$ & $\begin{array}{l}0.17 \\
0.91 \\
0.06\end{array}$ \\
\hline $\begin{array}{l}\text { Vascular access } \\
\text { RRA } \\
\text { LRA } \\
\text { RFA } \\
\text { LFA }\end{array}$ & $\begin{aligned} 15 & (25.9) \\
2 & (3.4) \\
36 & (62.1) \\
5 & (8.6)\end{aligned}$ & $\begin{array}{l}10(50) \\
0(0) \\
9(45) \\
1(5)\end{array}$ & $\begin{array}{l}0.04 \\
0.40 \\
0.18 \\
0.60\end{array}$ & $\begin{array}{c}16(34.8) \\
1(2.2) \\
24(52.2) \\
5(10.9)\end{array}$ & $\begin{aligned} 9 & (28.1) \\
1 & (3.1) \\
21 & (65.6) \\
1 & (3.1)\end{aligned}$ & $\begin{array}{l}0.53 \\
0.79 \\
0.23 \\
0.20\end{array}$ \\
\hline
\end{tabular}

b.c. - balloon catheter; BMS - bare-metal stent; Cx - circumflex branch; DEB - drug-eluting balloon; DES - drug-eluting stent; Dg - diagonal branch; IVUS - intravascular ultrasound; LAD - left anterior descending branch; LFA - left femoral artery; LMCA - left main coronary artery; LRA - left radial artery; Mg - marginal branch; PCI - percutaneous coronary intervention; POBA - plain-old balloon angioplasty; RCA - right coronary artery; RFA - right femoral artery; RRA - right radial artery. 
Table 4. Procedure-related complications

\begin{tabular}{|c|c|c|c|c|c|c|}
\hline \multirow{2}{*}{ Variable } & \multicolumn{2}{|c|}{ Gender } & \multirow{2}{*}{$p$-value } & \multicolumn{2}{|c|}{ Age [years] } & \multirow{2}{*}{$p$-value } \\
\hline & male & female & & $<75$ & $\geq 75$ & \\
\hline Overall rate & $13(18.3)$ & $12(46.1)$ & 0.005 & $10(16.1)$ & $15(42.8)$ & 0.003 \\
\hline$A D$ & $3(4.2)$ & $2(7.7)$ & 0.49 & $3(4.8)$ & $2(5.7)$ & 0.85 \\
\hline CS & $1(1.4)$ & $3(11.5)$ & 0.02 & $2(3.2)$ & $2(5.7)$ & 0.55 \\
\hline MI & $2(2.8)$ & $1(3.8)$ & 0.79 & $2(3.2)$ & $1(2.8)$ & 0.91 \\
\hline Death & $0(0)$ & $1(3.8)$ & 0.09 & $0(0)$ & $1(2.8)$ & 0.18 \\
\hline Bleeding & $1(1.4)$ & $2(7.7)$ & 0.11 & $1(1.6)$ & $2(5.7)$ & 0.27 \\
\hline Hematoma & $1(1.4)$ & $2(7.7)$ & 0.11 & $0(0)$ & $3(8.6)$ & 0.02 \\
\hline Rotawire disruption & $0(0)$ & $1(3.8)$ & 0.09 & $0(0)$ & $1(2.8)$ & 0.18 \\
\hline Arrhythmias & $2(2.8)$ & $0(0)$ & 0.38 & $1(1.6)$ & $1(2.8)$ & 0.67 \\
\hline Allergic reaction & $1(1.4)$ & $0(0)$ & 0.54 & $1(1.6)$ & $0(0)$ & 0.45 \\
\hline Slow flow & $1(1.4)$ & $0(0)$ & 0.54 & $0(0)$ & $1(2.8)$ & 0.18 \\
\hline $\mathrm{CIN}$ & $1(1.4)$ & $0(0)$ & 0.54 & $0(0)$ & $1(2.8)$ & 0.18 \\
\hline
\end{tabular}

AD - arterial dissection; CIN - contrast-induced nephropathy; CS - cerebral stroke; MI - myocardial infarction.

\section{Clinical outcomes and follow-up}

Follow-up was completed among the overall study group in $78(80.4 \%)$ patients included into the study. During the mean time to the MACCE or follow-up without MACCE (695 \pm 560 days), MACCEs occurred in 26 patients (33.7\%). Among the MACCEs, there were 14 deaths (53.8\%), 4 reinterventions (15.4\%), 5 MIs (19.2\%), 2 CS/TIA cases (7.7\%), and 1 coronary artery by-pass grafting (CABG) operation (3.8\%).

The frequency of MACCEs was greater among females compared to males, however, without statistical significance $(45.4 \%$ vs $28.6 \%, \mathrm{p}=0.32)$. This was mainly due to greater rate of reinterventions $(9.1 \%$ vs $3.6 \%, \mathrm{p}=0.7)$ and deaths $(27.3 \%$ vs $14.3 \%, \mathrm{p}=0.37)$. Also, the MACCEs rate was greater in patients older than 75 compared to those younger than 75 ( $42.8 \%$ vs $28.6 \%, \mathrm{p}=0.38$ ), and this was mainly the consequence of greater rate of deaths among older patients $(35.7 \%$ vs $8 \%, \mathrm{p}=0.04)$. This is presented in Table 5 .
The Kaplan-Meier survival curve comparison between males and females confirmed that, despite the fact that the frequency of MACCEs was higher in women, there was no significant difference between those 2 groups $(p=0.07)$. This is presented in Fig. 2.

Similarly, the greater rate of MACCEs in patients older than 75 compared to those younger did not meet statistical significance in comparison of the Kaplan-Meier survival curves $(\mathrm{p}=0.36)$. This is presented in Fig. 3 .

\section{Predictors of clinical outcomes}

Univariate analysis of several selected factors revealed that among them, statistically significant relationships with MACCEs were achieved for the maximal burr diameter $(\mathrm{p}=0.03)$, the occurrence of procedure-related complications $(\mathrm{p}=0.015)$, the mean EUROSCORE II value $(\mathrm{p}=0.02)$, and borderline relationship with obesity $(p=0.052)$. Considering the fact that age and gender were

Table 5. Study endpoints and duration of follow-up in selected groups of patients

\begin{tabular}{|c|c|c|c|c|c|c|}
\hline \multirow{2}{*}{ Variable } & \multicolumn{2}{|c|}{ Gender } & \multirow{2}{*}{$\mathrm{p}$-value } & \multicolumn{2}{|c|}{ Age [years] } & \multirow{2}{*}{$p$-value } \\
\hline & male & female & & $<75$ & $\geq 75$ & \\
\hline Completed follow-up & $56(78.9)$ & $22(84.6)$ & 0.90 & $50(80.6)$ & $28(80)$ & 0.88 \\
\hline Mean time of follow-up [days] & $727 \pm 536$ & $614 \pm 625$ & 0.18 & $677 \pm 541$ & $726 \pm 603$ & 0.80 \\
\hline MACCE & $16(28.6)$ & $10(45.4)$ & 0.32 & $14(28.6)$ & $12(42.8)$ & 0.38 \\
\hline Mean time to MACCE [days] & $638 \pm 573$ & $324 \pm 435$ & 0.09 & $574 \pm 634$ & $451 \pm 415$ & 0.95 \\
\hline $\mathrm{Re}-\mathrm{PCl}$ & $2(3.6)$ & $2(9.1)$ & 0.70 & $4(8)$ & $0(0)$ & 0.55 \\
\hline Death & $8(14.3)$ & $6(27.3)$ & 0.37 & $4(8)$ & $10(35.7)$ & 0.04 \\
\hline $\mathrm{CS} / \mathrm{TIA}$ & $1(1.8)$ & $1(4.5)$ & 0.85 & $1(2)$ & $1(3.6)$ & 0.90 \\
\hline Ml & $4(7.1)$ & $1(4.5)$ & 0.34 & $4(8)$ & $1(3.6)$ & 0.19 \\
\hline$C A B G$ & $1(1.8)$ & $0(0)$ & 0.90 & $1(2)$ & $0(0)$ & 0.88 \\
\hline
\end{tabular}

CABG - coronary artery by-pass grafting; CS - cerebral stroke; MACCE - major adverse cardiac and cerebrovascular events; MI - myocardial infarction; $\mathrm{PCl}$ - percutaneous coronary intervention; TIA - transient ischemic attack. 


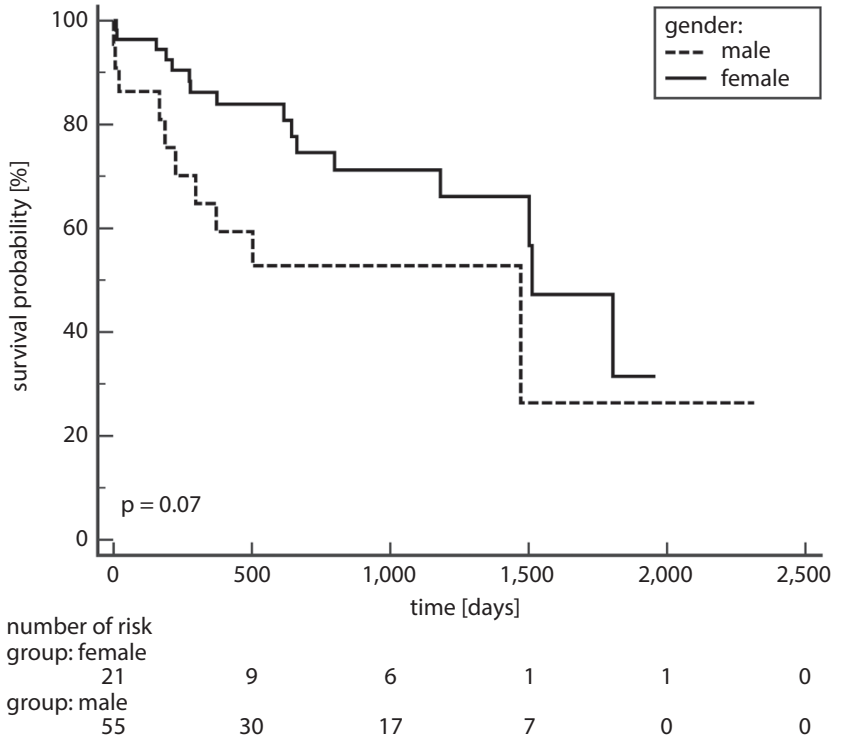

Fig. 2. The comparison of Kaplan-Meier survival curves according to gender

not among the factors associated with the occurrence of MACCE in the follow-up period, a multivariate analysis was not performed.

\section{Discussion}

The crucial finding of the presented analysis is that gender and age greater than 75 were not found to be significantly related to poorer clinical outcomes expressed as MACCE rate during the long-term follow-up period compared with survival curves. However, it should be noted after the analysis of individual components of MACCEs, for both women and patients in the age group above 75 , the incidence of deaths was higher, and in the case of age-related differences, mortality was statistically significantly greater in the group of older patients. The univariate analysis did not confirm the relationship of age and gender with MACCEs during the follow-up period. Instead, it revealed that the maximal burr diameter, EUROSCORE II value and the presence of procedure-related complications are associated with long-term clinical outcomes after RA. The relationship between the EUROSCORE II value and the length of the implanted stent was demonstrated in a previously published paper. ${ }^{15}$ The level of perforations in the presented study was higher $(3.1 \%)$ when compared to the published results based on large registries $(0.3-1.1 \%) .{ }^{16,17}$ However, coronary perforations observed in our analysis were not complicated by serious and life-threatening incidents, including cardiac tamponade and surgical treatment. They were treated successfully with an endovascular approach. Moreover, usually registries, including the Polish National Registry, underestimate the rate of procedure-related complications. ${ }^{18,19}$ The frequency of the overall procedure-related

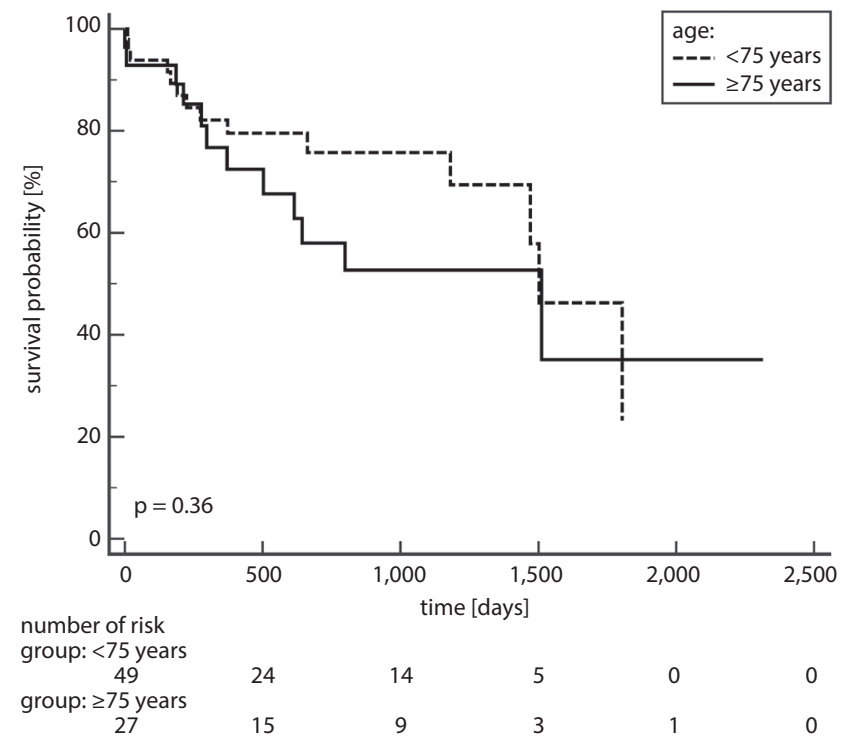

Fig. 3. The comparison of Kaplan-Meier survival curves according to the age of 75

complication rates was significantly greater in females and in the group of patients older than 75 . In the case of age, this was mainly due to the greater number of arterial dissections, cardiogenic shocks and MIs, while in the case of the female gender, arterial dissections, cardiogenic shocks, arterial perforations, and hematomas demanding blood transfusion or surgical treatment were more frequent.

Some published studies estimating the relationship between age and long-term clinical outcomes in patients with heavy calcified coronary artery lesions demonstrated that age is an independent risk factor of poorer clinical outcomes expressed as MACCE. ${ }^{6}$ However, most of them did not confirm a significant relationship between age and long-term clinical outcomes, and among the factors that have independent influence on the prognosis, they confirmed LVEF, diabetes, cardiogenic shock, acute coronary syndrome, kidney failure, SYNTAX score, neutrophil/lymphocyte ratio, burr to artery ratio, or prior PCIs. ${ }^{20-23}$ Most of these studies involved several hundreds of patients. Considering the various components of MACCE, AbdelWahab et al. demonstrated that age was an independent predictor of target lesion revascularization. ${ }^{20}$ Our study included only about 100 participants, which makes it one of the smaller studies among those discussed. As a consequence, the results are very susceptible to bias. One of the factors that certainly influenced the bias was the severe state of some patients qualified for RA at a young age for the present cohort of patients, which were the causes of MACCE occurrence during the hospital stay or shortly after discharge. Operators are less likely to perform heroic rotablation treatments in older patients with multiple risk factors, which undoubtedly influenced the artificial masking of mortality in the early periprocedural stage in patients over 75 . 
Moreover, we did not break down MACCE into individual components in order to assess the impact of age and gender in survival curve analysis on long-term treatment results due to an insufficient number of endpoints in individual groups of patients and the resulting high probability of error and bias. However, we performed such a comparison of study endpoints for both groups and showed almost twice as high mortality in the follow-up period in women and patients over the age of 75 , although this difference was statistically significant only for older patients. Nonetheless, in the case of women, this relationship can be explained by the higher mean value of the initial EUROSCORE II, which was mainly associated with a greater number of salvage mode procedures, greater mean age and more procedure-related complications. On the other hand, it should also be noted that women initially had a statistically significantly higher mean LVEF and they smoked cigarettes less frequently, which in some way influenced mortality balance and made it statistically non-significant. In the case of older age, the mortality rate was statistically significantly higher in the group of patients older than 75 , which was in line with other factors closely related to increased mortality as serum creatinine, GFR, mean SYNTAX score value, or mean EUROSCORE II value. In addition, the age of the patients and the aging of the body were also of great importance, as exemplified by the occurrence of deaths in patients above 90 years of age after a period of just over 200 days of the follow-up period. There are no publications attainable in the available literature that closely compare the long-term clinical outcomes of patients with severely calcified lesions of coronary arteries using RA. On the other hand, studies estimating the influence of individual factors on the results of treatment did not show that gender was related to treatment outcomes presented as MACCE prevalence. One of the few available studies on the treatment of similar coronary artery lesions, but undertaken with the use of another device, regards orbital atherectomy. ${ }^{7}$ This study was conducted on a relatively large group of patients including 458 participants. The incidence of MACCE did not differ significantly between the 2 groups and was $0.7 \%$ and $2.7 \%$, respectively, for women and men $(\mathrm{p}=0.14)$. Admittedly, the incidence of MACCE in this study was higher in men than women. One of the factors that could have an impact on the incidence of MACCE in this study was higher mean length and diameter of stents in men compared to women, which was shown to be related to the incidence of MACCE in the follow-up period in other studies, including those published by our center. ${ }^{15}$ On the other hand, in this study, the men were statistically significantly younger and less likely to have hypertension and hypercholesterolemia. Men were also insignificantly younger in our study, which certainly influenced the results.

Considering the relationship of procedure-related complications with gender and age, it turned out that the incidence of complications in both women and older individuals is greater. Already in a previously published publication by our center, we demonstrated on a large group of patients that the incidence of arterial perforations was related to age, and that age is an independent predictor of increased risk of their occurrence, ${ }^{18}$ which is in contrast, for example, to acute coronary syndromes that have not been shown to be significantly associated with the incidence of procedure-related complications. ${ }^{24}$ The increased rate of procedure-related complications is mainly caused by calcifications in the coronary arteries, which predispose to typical complications of RA such as dissections or perforations. ${ }^{16,25}$ Previously published studies on the association of gender with periprocedural complications treated with PCI indicate a greater susceptibility of women to procedure-related complications. ${ }^{26}$ This trend also seems to be sustained for PCI with RA.

\section{Conclusions}

The negative relationship of age and gender with clinical outcomes expressed as increased rate of MACCEs in elderly patients treated with PCI and RA does not have as much impact as in the case of patients in other age groups, which should encourage operators to reduce their concerns in the treatment of patients over 75 years of age with rotablation.

\section{Study limitations}

The results of the presented work should be received with great caution, because the influence of age on poorer results in the follow-up period seems to be evident in all groups of patients treated with percutaneous coronary angioplasty, although the purpose of this article is to show that wellprepared and stable patients among the elderly may achieve comparable benefits from the use of rotablation compared to younger patients. In the current work, apart from a very small group of patients as for this type of treatment and a relatively large number of patients lost in the follow-up period, there are a number of factors that artificially mask the actual impact of age and sex on long-term treatment results. One of the main factors is undoubtedly the greater willingness of the use of rotablation in the salvage mode, especially in younger and promising patients, compared to the older and less promising individuals burdened with many concomitant diseases, including cancer, heart failure and others, which artificially increased the number of deaths in the early periprocedural stage in patients under 75 years of age.

\section{ORCID iDs}

Rafał Januszek (D) https://orcid.org/0000-0002-6591-1919 Artur Pawlik (i) https://orcid.org/0000-0001-6234-7243 Bartłomiej Staszczak (D) https://orcid.org/0000-0002-5368-515X Magdalena Jędrychowska (D) https://orcid.org/0000-0001-7155-6565 Jerzy Bartuś (D) https://orcid.org/0000-0002-4288-3724

Jacek Legutko (D) https://orcid.org/0000-0002-2945-3674 Dariusz Dudek (D) https://orcid.org/0000-0002-3189-2414 Andrzej Surdacki (D) https://orcid.org/0000-0001-7949-3140 Stanisław Bartuś (D) https://orcid.org/0000-0003-3180-8865 


\section{References}

1. Creditor MC. Hazards of hospitalization of the elderly. Ann Intern Med. 1993;118(3):219-223.

2. Walter LC, Brand RJ, Counsell SR, et al. Development and validation of a prognostic index for 1-year mortality in older adults after hospitalization. JAMA. 2001;285(23):2987-2994.

3. Alexander KP, Newby LK, Cannon CP, et al. Acute coronary care in the elderly, part I. Non-ST-segment-elevation acute coronary syndromes: A scientific statement for healthcare professionals from the American Heart Association Council on Clinical Cardiology: In collaboration with the Society of Geriatric Cardiology. Circulation. 2007;115(19):2549-2569.

4. Vliegenthart R, Oudkerk M, Hofman A, et al. Coronary calcification improves cardiovascular risk prediction in the elderly. Circulation. 2005;112(4):572-577.

5. Barbato $E$, Carrié $D$, Dardas $P$, et al. European expert consensus on rotational atherectomy. Eurolntervention. 2015;11(1):30-36.

6. Okai I, Dohi T, Okazaki S, et al. Clinical characteristics and long-term outcomes of rotational atherectomy: J2T Multicenter Registry. Circ J. 2018;82(2):369-275.

7. Lee MS, Shlofmitz E, Mansourian P, et al. Gender-based differences in outcomes after orbital atherectomy for the treatment of de novo severely calcified coronary lesions. J Invasive Cardiol. 2016;28(11): 440-443.

8. Thygesen K, Alpert JS, Jaffe AS, et al. Third universal definition of myocardial infarction. Eur Heart J. 2012;33(20):2551-2567.

9. Ellis SG, Ajluni S, Arnold AZ, et al. Increased coronary perforation in the new device era. Incidence, classification, management, and outcome. Circulation. 1994;90(6):2725-2730.

10. Ponikowski P, Voors AA, Anker SD, et al. 2016 ESC Guidelines for the diagnosis and treatment of acute and chronic heart failure: The Task Force for the diagnosis and treatment of acute and chronic heart failure of the European Society of Cardiology (ESC). Developed with the special contribution of the Heart Failure Association (HFA) of the ESC. Eur Heart J. 2016;37(27):2129-2200.

11. Mehran R, Nikolsky E. Contrast-induced nephropathy: Definition, epidemiology, and patients at risk. Kidney Int Suppl. 2006;100:S11-15.

12. Rogers JH, Lasala JM. Coronary artery dissection and perforation complicating percutaneous coronary intervention. J Invasive Cardiol. 2004;16(9):493-499.

13. Barbato $E$, Carrié $D$, Dardas $P$, et al. European expert consensus on rotational atherectomy. Eurolntervention. 2015;11(1):30-36.

14. Antman EM, Cohen M, Bernink PJ, et al. The TIMI risk score for unstable angina/non-ST elevation MI: A method for prognostication and therapeutic decision making. JAMA. 2000;284(7):835-842.
15. Bartuś S, Januszek R, Legutko J, et al. Long-term effects of rotational atherectomy in patients with heavy calcified coronary artery lesions: A single-centre experience. Kardiol Pol. 2017;75(6):564-572.

16. Cockburn J, Hildick-Smith D, Cotton J, et al. Contemporary clinical outcomes of patients treated with or without rotational coronary atherectomy: An analysis of the UK central cardiac audit database. Int J Cardiol. 2014;170(3):381-387.

17. Iannaccone M, Piazza F, Boccuzzi GG, et al. ROTational AThErectomy in acute coronary syndrome: Early and midterm outcomes from a multicentre registry. Eurolntervention. 2016;12(12):1457-1464.

18. Januszek R, Siudak Z, Dziewierz A, et al. Predictors of in-hospital effectiveness and complications of rotational atherectomy (from the ORPKI Polish National Registry 2014-2016). Catheter Cardiovasc Interv. 2018;92(4):E278-E287.

19. Januszek R, Dziewierz A, Siudak Z et al. Chronic obstructive pulmonary disease and periprocedural complications in patients undergoing percutaneous coronary interventions. PLoS One. 2018;13(10): e0204257.

20. Abdel-Wahab M, Baev R, Dieker P, et al. Long-term clinical outcome of rotational atherectomy followed by drug-eluting stent implantation in complex calcified coronary lesions. Catheter Cardiovasc Interv. 2013;81(2):285-291.

21. Édes IF, Ruzsa Z, Szabó G, et al. Clinical predictors of mortality following rotational atherectomy and stent implantation in high-risk patients: A single center experience. Catheter Cardiovasc Interv. 2015; 86(4):634-641.

22. Cuenza LR, Jayme AC, Khe Sui JH. Clinical outcomes of patients undergoing rotational atherectomy followed by drug-eluting stent implantation: A single-center real-world experience. Heart Views. 2017;18(4): $115-120$.

23. Eftychiou C, Barmby DS, Wilson SJ, et al. Cardiovascular outcomes following rotational atherectomy: A UK multicentre experience. Catheter Cardiovasc Interv. 2016;88(4):546-553.

24. Januszek R, Siudak Z, Dziewierz A, et al. Bailout rotational atherectomy in patients with myocardial infarction is not associated with an increased periprocedural complication rate or poorer angiographic outcomes in comparison to elective procedures (from the ORPKI Polish National Registry 2015-2016). Postepy Kardiol Interwencyjnej. 2018;14(2):135-143.

25. Fitzgerald PJ, Ports TA, Yock PG. Contribution of localized calcium deposits to dissection after angioplasty: An observational study using intravascular ultrasound. Circulation. 1992;86(1):64-70.

26. Serruys PW, Cavalcante R, Collet $C$, et al. Outcomes after coronary stenting or bypass surgery for men and women with unprotected left main disease: The EXCEL Trial. JACC Cardiovasc Interv. 2018;11(13): 1234-1243. 